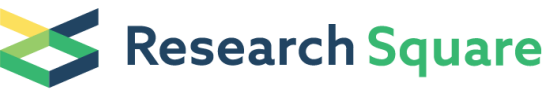 \\ Preprints are preliminary reports that have not undergone peer review. \\ They should not be considered conclusive, used to inform clinical practice, or referenced by the media as validated information.
}

\section{Aneuploid circulating tumor endothelial cells (CTECs) affect the detection of CTC, but they are fundamentally different}

\section{Yuanyuan Lei}

national cancer center/national clinical research center for cancer/cancer hospital

\section{Guochao Zhang}

national cancer center /national clinical research center for cancer/cancer hospital

Chengming Liu

national cancer center/national clinical research center for cancer/cancer hospital

\section{Zhiliang Lu}

national cancer center/national clinical research center for cancer/cancer hospital

\section{Jianbing Huang}

national cancer center/national clinical research center for cancer/cancer hospital

\section{Chaoqi Zhang}

national cancer center/national clinical research center for cancer/cancer hospital

\section{Ruochuan Zang}

national cancer center/national clinical research center for cancer/cancer hospital

\section{Yun Che}

national cancer center/national clinical research center for cancer/cancer hospital

\section{Shuangshuang Mao}

national cancer center/national clinical research center for cancer/cancer hospital

\section{Lingling Fang}

national cancer center /national clinical research center for cancer/cancer hospital

\section{Xinfeng Wang}

national cancer center/national clinical research center for cancer/cancer hospital

\section{Sufei Zheng}

national cancer center/national clinical research center for cancer/cancer hospital

\section{Nan Sun}

national cancer center/national clinical research center for cancer/cancer hospital

Jie He ( $\square$ prof.jiehe@gmail.com )

National Cancer Center/National Clinical Center for Cancer/Cancer Hospital, Chinese Academy of Medical Sciences and Peking Union Medical College 
Keywords: Circulating tumor cells, circulating tumor endothelial cells, non-small cell lung cancer, liquid biopsy, tumor markers

Posted Date: April 29th, 2020

DOI: https://doi.org/10.21203/rs.3.rs-25021/v1

License: (c) (1) This work is licensed under a Creative Commons Attribution 4.0 International License. Read Full License 


\section{Abstract \\ Background}

Aneuploidy is an important criterion for tumor cell identification. Like CTCs (circulating tumor cells), many CTECs (circulating tumor endothelial cells) have chromosomal aneuploidy. Affected by tumor microenvironment and detection method, the only identification index CD31 may not be comprehensive enough, which greatly affects the accuracy of CTCs detection.

\section{Methods}

Here, we explored the number distribution, expression of surface tumor markers and chromosome ploidy differences of aneuploid CTECs and CTCs in a total of 98 peripheral blood from normal volunteers, benign nodules and lung cancer patients, trying to find the differences between the two and the clinical significance of CTEC.

\section{Results}

We found that CTCs were mainly dominated by triploid and hyperploid (pentaploid or above), The former was mainly small CTCs, and the latter was mainly large CTCs. While the aneuploid CTECs were mostly large tetraploid cells. Combined detection of total CTC and CTEC numbers helped identify benign and malignant nodules. Furthermore, tetraploid CTC and CTEC had more obvious advantages than other ploids in identifying benign and malignant nodules.

\section{Conclusions}

Our findings indicated that the traditional method of distinguishing tumor and non-tumor cells by aneuploidy was not accurate. The presence of aneuploidy CTECs greatly interfered with the detection of CTCs, but they were different form CTC in cell size, chromosome ploidy distribution and had certain clinical significance. Combining CTC and CTEC had better diagnostic advantages

\section{Introduction}

There are more than 700,000 new cases of lung cancer in China each year, accounting for $17 \%$ of all new cancer cases[1]. In the past 20 years, the incidence of lung cancer has continued to rise, with a five-year survival rate of about $16-18 \%$. Lack of early diagnosis and remote metastasis without warning are important factors in the low five-year survival rate of lung cancer. Therefore, it is especially important to find accurate markers for disease discovery and disease progression. 
Circulating tumor cells (CTCs) are a type of cells that are released into the blood from the primary or metastatic foci. They are scattered around the body with the blood circulation. When they encounter a suitable microenvironment, they can develop into micro-metastases. They are a very small, phenotypically and functionally diverse group of cells in the blood. The CTCs detection is now recognized as the most representative "liquid biopsy" technology, and the integrity and clinical significance of the various information it provides far exceeds other technologies. However, accurate detection of CTCs has largely limited its clinical application.

Unlike normal diploid cells, the chromosomes of cancerous cells undergo different degrees of variation, showing a state of aneuploidy. SE-iFISH technology can simultaneously separate tumor cells from peripheral blood by combining expression of tumor markers on the surface of tumor cells and chromosomal aneuploidy. However, we found in the previous period that there were still a large number of non-blood-derived circulating tumor endothelial cells (CTECs) in peripheral blood. These cells had normal diploid and aneuploid, but the endothelial marker CD31 was strongly positive, while aneuploid CTECs largely interferes with CTCs detection and their biological characteristics and clinical significance are unclear. During the process of tumor cells detaching from the primary tumor into the blood, the tumor microenvironment has undergone great changes, and the biological characteristics of the tumor cells have also changed. Aneuploid CTCs and CTECs are two cell populations that are distinguished only by CD31 positivity. Whether this classification is reasonable or whether there is a certain relationship between the two cells in the source needs further verification. Our study confirmed that there were a certain number of aneuploid CTCs and CTECs in normal humans, benign nodules, and patients with different stages of lung adenocarcinoma. We hypothesized that CTCs and CTECs were very different in terms of number, size, surface markers, and chromosomal aneuploidy.

In this article, we further expanded the number of samples, including early and late lung adenocarcinoma, benign nodules and normal volunteers, and further explored the biological differences between aneuploid CTCs and CTECs and their number distribution in the development of lung cancer. Analysis of surface tumor markers and changes in chromosome ploidy may provide new ideas for the diagnosis of lung adenocarcinoma. At the same time, subclass analysis of CTCs and CTECs can guide the direction of lung cancer targeted therapy

\section{Materials And Methods}

\section{Patients and samples}

All recruited subjects were from the Cancer Hospital of the Chinese Academy of Medical Sciences. Sample processing and preservation were done by the relevant staff of the thoracic surgery laboratory. The blood sample collection began on November 28, 2017 and ended in December 2019. A total of 98 fresh peripheral blood samples were included, including 18 healthy volunteers, 22 benign lung nodules, 34 early stage adenocarcinoma patients and 24 advanced lung adenocarcinoma. Normal blood specimens came from healthy volunteers recruited by the thoracic surgery laboratory. The inclusion 
criteria were between 45-85 years old, no precancerous lesions, no tumor history, and a family history of no tumor genetics. Specimens of benign nodules and early lung adenocarcinoma were obtained from patients undergoing surgery in thoracic surgery. Blood samples were collected for enriching and identifying for circulating cells one day before surgery. statistics on specimen categories based on postoperative pathology; Patients with advanced lung adenocarcinoma were from the Department of Oncology, and the inclusion criteria were untreated (including radiotherapy and chemotherapy) or newly diagnosed patient.

When collecting peripheral blood of patients, in order to avoid contamination of epithelial cells, the first $2 \mathrm{ml}$ blood sample were discarded or blood samples were taken after routine blood sampling of patients. Blood samples were taken from ACD anticoagulated blood vessels. After collecting the blood, immediately mix the head and tail 8 times. Store at room temperature and protected it from light. Try to process within 24 hours, not more than $48 \mathrm{~h}$.

The study was approved by the Ethics Committee of the Cancer Hospital of the Chinese Academy of Medical Sciences before the study. All patients were given informed consent before blood collection.

\section{Subtraction Enrichment Of Human Peripheral Blood}

The CTC enrichment kit was from Cytointelligen, and the procedure was performed according to the procedures specified in the kit. After collecting the blood sample, the blood collection tube was leveled, and the head and tail were mixed upside down, and then centrifuged at room temperature for 15 minutes $(200 \times \mathrm{g})$. Discard the supernatant to $5 \mathrm{~mm}$ above the brown-red precipitate and add CRC buffer to the blood vessel label to fill the tube to dilute the blood cells. Next, $3 \mathrm{ml}$ of the sample density separation liquid was added to a $50 \mathrm{ml}$ centrifuge tube, and the diluted blood cells were added to the upper layer of the sample density separation solution, and after trimming, the mixture was centrifuged at room temperature for 6 minutes $(350 \times \mathrm{g})$. After centrifugation, the sample was divided into three layers, and the intermediate transparent or yellow liquid and the upper yellow liquid were taken up to another new $50 \mathrm{ml}$ centrifuge tube. $300 \mathrm{ul}$ of the washed magnetic beads were added to the portion of the liquid and incubated on a shaker at room temperature for 20 minutes. Finally, the magnetic beads are removed by adsorption of a magnetic frame, and non-blood-derived cells (including tumor cells and endothelial cells) are enriched in the remaining liquid.

\section{Tumor Marker Immunostaining-chromosome Fluorescence In Situ Hybridization (TM-iFISH)}

The following reagents are derived from Cytointelligen. Take $100 \mathrm{ul}$ of the enriched cell solution, add $2 \mathrm{ul}$ of antigen repair buffer, mix gently and shake, and let stand for 10 minutes at room temperature. At the same time, the staining solution was configured. According to each slide, $200 \mathrm{ul}$ of blood cell analysis dilution +1 ul CD 45 staining solution $+1 \mathrm{ul} \mathrm{CD} 31$ staining solution + 1 ul vimentin staining solution; Use a 
sampler to gently mix and mix 10 times to fully mix the antibody. All the prepared mixture was added to the antigen-repaired cell sample and incubated for 20 minutes at room temperature. The stained cell sample was then washed by adding a CRC washing solution, centrifuged for 5 minutes $(500 \times \mathrm{g})$, and the supernatant was discarded to $100 \mathrm{ul}$. The cells were initially fixed by adding an equal volume of tissue fixative. Then dry overnight. The cells on the slide were fixed again the next day, and the fish probe was added for hybridization (hybridization conditions: denaturation at $76^{\circ} \mathrm{C}$ for 10 minutes; hybridization at $37^{\circ} \mathrm{C}$ for 3 hours). After the hybridization was completed, the coverslip was slowly removed with a buffer, and the sample washing area was added to wash the sample area again to reduce specific staining. Dry the cleaned slide specimen frame with a hair dryer, and add $10 \mathrm{ul}$ of DAPI dye solution to the center of the specimen frame, and cover the cover glass so that the dye solution was fully immersed in the entire specimen area. Immediately observe under a fluorescence microscope or preserve at $2-8{ }^{\circ} \mathrm{C}$ in the dark.

\section{Statistical Analyses}

Statistical analysis was done with Prism GraphPad software v8.0. All data are presented descriptively as means, medians, or proportions. Two-tailed Student's t-test was used for statistical comparison between groups; Pearson correlation was used to compare the correlation between the number of CTCs and CTECs. Statistical significance was defined as $p<0.05$.

\section{Results}

\section{Isolation and identification of aneuploid CTCs and CTECs}

Firstly, we removed the blood-derived cells such as red blood cells and white blood cells by centrifugation and phase-enrichment to achieve higher CTC enrichment efficiency, and then we borrowed the iFISH platform, which combine the immunofluorescence staining of tumor proteins (vimentin, KI67, PD-L1, etc.) with the detection of chromosomal aneuploidy. It can achieve the subclassification of CTCs with a higher sensitivity and specificity. The CTC identification criteria are as follows (Fig. 1A): nuclear DAPI+, CD45-, CD31-, chromosome 8 (CEP8) aneuploidy positive, and immunofluorescent staining CTC tumor markers positive or negative. Using this CTC sorting method, we successfully enriched and identified CTCs in peripheral blood of 98 patients (clinical characteristics are shown in Table 1). When screening aneuploid CTCs under a fluorescence microscope, we also found a large number of aneuploid endothelial cells (CTECs). These cells had the same degree of aneuploidy as tumor cells, but the endothelial cell marker CD31 was strongly positive (Fig. 1B)

\section{Distribution of total CTC and CTEC numbers in patients by stage}

We used the iFISH platform to enrich and identify CTCs and CTECs in 17 healthy volunteers, 22 benign pulmonary nodules, 34 early lung adenocarcinoma patients, and 22 advanced lung adenocarcinoma 
patients. We found that CTCs could be detected in the very early stages of lung cancer. Of the 34 early stage lung cancer patients, 29 cases detected the presence of CTCs, of which 17 cases had more than three CTCs. A certain number of CTCs could also be isolated in normal and benign nodule patients. This part of the results indicated that there was a certain background pollution in normal people and benign nodules (non-tumor cells of aging or apoptosis also had a certain aneuploidy, which was mistaken for tumor cells), but the number was mostly less than three, which indicated that CTCs still had certain advantages in identifying benign nodules and early stage lung cancer $(P=0.0119)$ (Fig. 2A). The number of CTCs in patients with advanced lung cancer was significantly increased. At the same time, the number of peripheral blood CTCs in patients with advanced lung adenocarcinoma was significantly higher than that in early lung cancer, benign nodules and normal controls, the difference was statistically significant $(P<0.0001)$ (Fig. 2A). In addition, we found some CTC-like aneuploidy circulating tumor endothelial cells (CTECs) during the experiment. Their quantitative changes in normal human, benign nodules and early and advanced lung cancer were consistent with CTCs (Fig. 2B). The number of CTECs in advanced patients was significantly higher than that of normal, benign nodules, and early stage lung cancer patients, and it had the same early diagnostic value $(P=0.0287)$ and tumor staging diagnostic value $(P=$ 0.0009 ) as CTCs. Since there are still normal diploid endothelial cells (CECs) in peripheral blood, this part of the cells is reported to have a certain staged diagnostic significance[2-6], and closely related to survival[7] and relapse[8] of cancer patient. We also counted the number of diploid CECs and found the number of diploid CECs in peripheral blood was very few, therefore we combined diploid CECs and aneuploid CTECs to observe their dynamic changes. The results showed that the total CEC score is not as good as the aneuploid CTECs in identifying early and late lung adenocarcinoma(Fig. 2C).Unexpectedly, we found that there was a certain correlation between CTCs and aneuploid CTECs in the peripheral blood, and there was a statistically significant difference(Fig. 2E) $(P=0.0003)$. CTCs and aneuploid CTECs are divided into two categories only because of the positive or negative CD31. As two independent and interrelated tumor markers, we suspected that the combined diagnosis of the two might have a better effect. The results showed that CTCs and aneuploid CTECs combined with a single marker had a greater advantage in distinguishing between benign and malignant nodules, statistical differences were more obvious (Fig. 2D) $(P=0.0069)$.

\section{Aneuploidy analysis of CTCs and CTECs}

Our study found that both CTCs and CTECs exhibited varying degrees of chromosome aneuploidy, namely, haploid, triploid, tetraploid, pentaploid and above, standard images are shown in Fig. 3A. The clinical relevance of different types of aneuploid CTCs has been demonstrated, for example, high-ploid CTCs has been shown to be associated with tumor resistance and relapse[9, 10]. However, the clinical significance of aneuploid CTECs are still unclear.

We found that these sorted CTC chromosomes 8 had a certain pattern. In all normal human, benign nodules, patients with early-stage or late-stage lung cancer, chromosomal abnormalities were predominantly polyploid (the most common are triploid and pentaploid), and only a few are haploid(Fig. 3B and 3D) (due to the very small number of haploids, there was no statistical subtype), 
which was in line with the literature reports that the polyploid genetic material was relatively abundant, conducive to the development of malignant biological behavior of cells. the vast majority of CTCs in patients with benign nodules were triploid, but in patients with cancer, especially in patients with advanced stages (Fig. 3B and 3D), the pentaploid begun to increase, explaining that the increase in genetic material was likely to increase the degree of malignancy. Although CTECs were also dominated by triploid and pentaploid, pentaploid account for the vast majority of patients, especially in advanced patients (Fig. 3C and 3E).

\section{Distribution of CTC and CTEC with different size in patients}

Peripheral blood CTCs and CTECs vary in size. The definition of large and small cells is generally based on white blood cells (5um), cells larger than 5um are defined as large cells, and cells smaller than $5 \mathrm{~cm}$ are defined as small cells, and the standard image is shown in Fig. 4A. The biological and clinical significance of CTCs are different. For example, small CTCs has been reported to be significantly associated with recurrence and disease-free survival (DFS) of liver cancer[10]. However, the correlation between the size of CTECs and its clinical significance is still unclear. Therefore, we separately calculated the distribution of size of CTCs and CTECs in patients with different stages of cancer. On average, except for small CTECs, both large and small CTCs and large CTECs had the effect of distinguishing between benign and malignant nodules and staging (Fig. 4B-4E). But compared to other subtypes, large CTCs had an advantage in identifying early and late lungs(Fig. 4B) $(P<0.0001)$, while large CTECs were more able to distinguish between benign nodules and early stage lung cancer patients $\square$ Fig. $4 D)(P=0.0085)$. In comparison, small CTECs were not only small in number, but also cannot do good and bad in prediction and staging diagnosis (Fig. 4E). But when we compared the proportion of large CTCs and large CTECs in different patient populations, we found that there was no difference in the proportion of total circulating cells in the two sub-categories, especially large CTCs, in four different populations (Fig. 4F)

\section{Chromosome ploidy distribution of different size of CTCs and CTECs}

Since chromosomal ploidy of CTCs and CTECs subclasses with different sizes have certain clinical significance, we next analyzed the chromosomal ploidy differences of large and small cells. We observed a significant difference in the cell size of different ploidy numbers (Fig. 5A-5D). According to the standard of 5um in diameter, CTCs were divided into large cells and small cells, and small cells were mainly triploid, while large cells mostly were pentaploid. The number of tetraploid was between that of triploid and pentaploid, and its size (large or small) was not much different (Fig. 5A and 5B), but the proportion of tetraploid in large CTECs was significantly higher than that in small CTECs (Fig. 5C and 5D). However, as shown in Fig. 4, the large cells were more significant than small cells in staging diagnosis between normal humans, benign nodules, and lung cancer.

\section{Subclass analysis of CTCs and CTECs based on chromosomal ploidy and cell size combination}


To further understand the significance of the various subtypes of CTCs and CTECs in the early diagnosis and staging of lung cancer, we divided CTCs and CTECs respectively into 24 subgroups based on cell size, chromosome ploidy and four stages (normal, benign, early, and late) of tumor development for separate analysis (Fig. 6A-6D).

Among the large CTCs, all the triploid, tetraploid and pentaploid could distinguish between early and late patients $(P<0.05)$, but only the pentaploid could identify benign nodules and early patients (Fig. $6 \mathrm{~A})$. And compared to the other two ploidy, the ability of the pentaploid to distinguish between early and late patients was stronger $(P<0.001)$. For small CTCs, tetraploid had obvious early diagnostic significance $(P<$ 0.001), while pentaploid had no advantage in both early diagnosis and staged diagnosis. However, because tetraploids accounted for only a small proportion of the entire CTC population, it might affect the sensitivity of its clinical application. When we combined the large CTCs and the small CTCs to reduce the subgroups, the sub-categories obtained after the analysis were similar in the distribution of the large CTCs in the different stages (Fig. 6A and 6E). Current reports on CTC ploidy tend to combine tetraploid and pentaploid into a subgroup, collectively referred to as polyploid. Compared to triploids, polyploids do find some clinical significance, such as related to metastasis, recurrence and drug resistance[9].

Therefore, we also tried to combine triploid and tetraploid into a subclass, namely, polyploid, and then compare the significance of this new subclass in the diagnosis of tumor staging. It was found that for large CTCs, the polyploid subclass after the combination had no obvious advantage in distinguishing between benign and malignant nodules and distinguishing between early and late patients compared with the single subclass before the merger (Fig. S1A). Moreover, once merged, the ability of small CTCs in identifying benign and malignant nodules was lower than before (Fig. S1B).

For CTECs, we did the same analysis. Large pentaploid CTECs could simultaneously identify benign and malignant nodules and classify for patients with early and late stage tumors (Fig. 6B). Small triploid CTECs could distinguish between early and late patients, but could not identify benign nodules and early patients (Fig. 6D). But after the combination of large and small CTECs, both tetraploid and pentaploid had certain clinical significance. The pentaploid was only meaningful for early and late stage patients, and tetraploid could distinguish between benign nodules and early lung cancer patients (Fig. 6F). When we combined the tetraploid and pentaploid of CTECs into a polyploid subclass, small polyploid CTECs could not stage clinical patients (Fig. S1D). And large CTECs had the same effect as before the merger, that is, only pentaploid was meaningful, and could distinguish between benign nodules and early patient or between early and late patients (Fig. S1C).

Based on the results of CTCs and CTECs, we found that for CTCs, the most advantageous one for identifying benign nodules and early patients was tetraploid small CTCs; the most appropriate for identifying early and late patients was the pentaploid large CTCs; For CTECs, the sub-class of best identifying benign nodules and early patients was the combination of large and small tetraploid cells or large polyploid cells. But the best choice for distinguishing between early and late patients was large pentaploid CTECs or large polyploid (tetraploid and pentaploid) CTECs. Finally, comparing CTCs and 
CTECs as a whole, whether in differentiating of benign and malignant nodules or of early and late lung cancer, CTCs were slightly more advantageous than CTECs.

\section{CTEC was not a senescent or apoptotic cell, but an active cell population}

In principle, endothelial cells are non-tumor cells, and the genetic material is relatively stable, while CTEC chromosomes exhibit different degrees of copy number variation, similar to the malignant transformation process of tumor cells. However, because these cells are strongly positive for CD31, although they have many similarities with tumor cells, they are classified as a column of endothelial cells. We suspected that these CTECs that entered the blood were all aging or impending apoptosis, therefore they showed chromosomal variation. However, we had discovered some new phenomena, such as the phenomenon of cell division in CTECs isolated from peripheral blood (Fig. 7A), and CTECs could be clustered like tumor cells(Fig. 7B) or grouping with other types of cells such as white blood cells(Fig. 7C) and tumor cells, in line with the literature reports[11]. The binding of CTCs to leukocytes can promote their own malignant phenotype[12], but the clinical significance of CTECs and leukocyte or tumor cell agglomeration is still unclear. At the same time, some CTECs could also express tumor markers such as vimentin (Fig. 7D). These phenomena together indicated that aneuploid CTECs could indeed exist as a separate active cell population like a tumor cell, rather than simply shed cells that fall into the blood after aging or death. But whether CTECs have potential malignant behavior and its true biological differences with CTCs requires further cellular and molecular experiments to validate, and the future will help to understand these issues with single-cell sequencing.

\section{Discussion}

In this article, we explored the biological characteristics of aneuploid CTCs and CTECs and their dynamic changes at different stages of lung cancer development based on chromosome 8 mutatio. We found that there were some similarities between the distribution and chromosomal changes of CTCs and aneuploidy CTECs. In terms of quantitative changes, both CTCs and aneuploid CTECs could be used as good indicators of lung adenocarcinoma staging; In chromosome ploidy variation, tetraploid small CTCs had advantages in identifying early and benign nodules, for tetraploid Small CTCs only occurred in patients with tumors; While pentaploid large CTCs could better distinguish early patient and late patient. CTECs were mainly composed of pentaploid large cells. The main advantage lied in the identification of early and advanced lung cancer.

Human blood is rich in biological information, and liquid biopsy can capture cancerous information at an early stage and prevent tumor progression as early as possible. Common liquid biopsy indicators are cfDNA, RNA, miRNA[13], and proteins (free proteins or proteins contained in CTCs, exosomes[14] or platelets[15]. cfDNA is commonly used to detect gene mutations, and is suitable for drug resistance and efficacy evaluation, but the blood contains a high abundance of non-tumor-derived cfDNA[16]; RNA is low in blood and not stable; Protein-based markers generally respond to disease progression stages 
depending on the level of content, but provide limited information that does not reflect changes in gene levels and tumor heterogeneity. Unlike fragmented nucleic acid and protein markers, aneuploid CTCs and CTECs together form a pair of "cell-type circulating tumor markers" that are biologically active and rich in a range of tumor markers and complete genetic information. The two functions are different and complement each other and play an important role in the occurrence and development of tumors.

The presence of tumor cells by chromosomal aneuploidy detection (FISH) has been recognized by the pathology departments of major hospitals. The use of chromosome 8 probe (CEP8) for the diagnosis of lung cancer, colorectal cancer, stomach cancer and liver cancer has been reported in many literatures. The iFISH technique, which combines FISH technology with cellular immunofluorescence staining, has been applied to CTC detection of various cancers. For example, Jing Zhang et al found that most of the newly diagnosed nasopharyngeal carcinoma patients could detect triploid CTCs, but polyploid ( $\geqq 5$ pentaploid) in most patients(66.7\%)with recurrence and metastasis increased significantly; They also validated that the copy number of chromosome 8 was closely related to chemotherapy efficacy and drug resistance, during which tetraploid CTCs were most sensitive to chemotherapy, while triploid CTCs were most relevant to drug resistance[17]. Polyploid chromosome 8 is also associated with tumor resistance and recurrence in breast cancer, gastric cancer[18], and liver cancer. Therefore, a more accurate sub-class analysis of CTCs based on chromosome 8 aneuploid brings new breakthroughs in the diagnosis and treatment of tumors.

Another important focus of the article is the aneuploid CTECs. Since 2004, the Japanese Kyoko Hida team has discovered in mouse tissue for the first time the phenomenon of DNA abnormalities in endothelial cells in tumor tissues[19]. Then in 2009, they found that in human kidney cancer tissues, approximately $22-58 \%$ of endothelial cells had aneuploidy of chromosomes 7 and 8[20]. Moreover, unlike normal stable diploid cells, the degree of karyotype variation of this aneuploid endothelial cell increased with the number of passages in vitro, that is, in the tumor microenvironment, tumor endothelial cells can obtain cytogenetic abnormalities[20]. They later did research to speculate that the production of aneuploid endothelial cells was likely caused by hypoxic conditions in the tumor microenvironment[21]. However, the discovery of aneuploid CTECs in the peripheral blood circulation was only unexpectedly discovered during the detection of CTCs after the rise of iFISH technology. First reported in 2017[11]. In our study, aneuploid CTEC does have obvious enrichment in peripheral blood. At different stages of lung cancer development and progression, its number shows dynamic changes, and it is obviously enriched in advanced patients, which has certain clinical reference significance, similar to the previous researches exploring the distribution trend of circulating endothelial cells[22, 23]. However, previous studies were mainly focused on total CEC and did not distinguish between aneuploid and normal diploids in detail. We separately analyzed aneuploid cells and diploid cells and found that the number of aneuploid CECs was much higher than that of normal diploid CECs. Whether they are detached from the aneuploid endothelial cells of the primary tumor is still unclear, and even because of the specificity of lung cancer tissue (nonspecific fluorescence), we cannot explore whether there is a different part of lung cancer tissue with aneuploid endothelial cells by traditional immunofluorescence, limiting the development of research. Tumor tissue-associated endothelial cells (TEC) have been proven to promote tumor development in all 
aspects[24, 25], but the role of aneuploid CECs in peripheral blood in the development of tumors needs further study.

During the process of tumor cells detaching from the primary tumor into the blood, the tumor microenvironment has undergone great changes, and the biological characteristics of the tumor cells have also changed. Aneuploid CTCs and CTECs are two cell populations that are distinguished by CD31 positivity or not, but they have similar numbers in different stages of lung cancer development, and the chromosome ploidy distribution is similar. At the same time, an article first reported in 2019 that aneuploid CTECs, like CTCs, can also express a series of tumor markers, such as HER2, PD-L1, EpCAM and stem cell marker CD44v6[26]. Simultaneous detection of PD-L1-positive aneuploid CTEC, CTC and other tumor markers brings great convenience to screening patients for immunotherapy[27]. Moreover, we observed aneuploidy CTECs rarely expressed the interstitial marker vimentin, while this indicator has a strong positive rate in the blood vessels. Then whether this classification method is reasonable or whether there may be some connection between the two kinds of cells in the source? It is probably a kind of transformation of cells just in the process of entering the blood. This conjecture needs further verification.

This study also has some shortcomings, for example, a small amount of CTCs and aneuploid CTECs were also found in normal and benign nodules. These two types of cells need to be further expanded in the early diagnosis of lung cancer to find a suitable cut off value. CTCs and aneuploid CTECs found in normal and benign nodules may be cells of chromosomal variation caused by inflammation and aging. This part of CTCs can be classified as "non-authentic" CTCs, and the degree of biological malignancy may be far less than the "true" CTCs, it may disappear by the autoimmune system at any time, so it is especially important to dynamically detect the number of such cells. The rise of future single-cell sequencing technology promises to find a marker that truly distinguishes between "truth" and "false" CTCs, making detection more precise.

\section{Conclusions}

There are significant differences in the number, size, surface markers and chromosomal aneuploidy between aneuploid and CTCs and CTECs in patients with lung adenocarcinoma at different stages. Both types of cells can play a certain role in the diagnosis and treatment of lung cancer. The future direction of development will be based on these biological characteristics for sub-class analysis of aneuploid CTCs and CTECs, guiding in-depth mechanism research and targeted therapy.

\section{Abbreviations}

CTCs: circulating tumor cells $₫$ CTECs: circulating tumor endothelial cells

\section{Declarations}




\section{Availability of data and materials}

The analyzed data sets generated during the study are available from the corresponding author on reasonable request.

\section{Abbreviation}

CTCs: circulating tumor cells $₫$ CTECs: circulating tumor endothelial cells

\section{Acknowledgments}

We thank Peter Lin for the excellent technical assistance.

\section{Funding}

This work was supported by the National Natural Science Foundation of China, CAMS Innovation Fund for Medical Sciences (2017-I2M-1-005, 2016- I2M-1-001 ), the National Key Research and Development Program of China (grant numbers 2016YFC1303201, 2016YFC0901400), the National Natural Science Foundation of China(81802299, 81502514),the Fundamental Research Funds for the Central Universities (3332018070), and the National Key Basic Research Development Plan (2018YFC1312105).

\section{Author information}

\section{Affiliations}

National cancer center/National clinical research center for cancer/Cancer hospital, CHINA

Yuanyuan Lei, Guochao Zhang, Chengming Liu, Zhiliang Lu, Jianbing Huang, Chaoqi Zhang, Ruochuan Zang, Yun Che, Shuangshuang Mao, Lingling Fang, Xinfeng Wang, Sufei Zheng, Nan Sun and Jie He

\section{Contributions}

YYL, NS, and JH were responsible for the study concept and design, acquisition, analysis and interpretation of data, and drafting the manuscript. GCZ, CML, LLF, CQZ and RCZ were responsible for collection of samples. ZLL, JBH, XFW and SFZ were responsible for processing specimen. YC and SSM were responsible for proofreading.

\section{Corresponding author}




\section{Ethics declarations}

\section{Ethics approval and consent to participate}

Informed consent was obtained, in accordance with the Declaration of Helsinki. The study was approved by the Ethics Committee of the Cancer Hospital of the Chinese Academy of Medical Sciences before the study. All patients were given informed consent before blood collection.

\section{Consent for publication}

Not applicable.

\section{Competing interests}

The authors declare that they have no competing interests.

\section{References}

1. Chen W, et al. Cancer statistics in China, 2015. CA Cancer J Clin. 2016;66(2):115-32.

2. De Biasi S, et al. High speed flow cytometry allows the detection of circulating endothelial cells in hemangioblastoma patients. Methods. 2018;134-135:3-10.

3. De Sanctis F, et al. The dark side of tumor-associated endothelial cells. Semin Immunol. 2018;35:3547.

4. Cauley CE, et al. Circulating Epithelial Cells in Patients with Pancreatic Lesions: Clinical and Pathologic Findings. J Am Coll Surg. 2015;221(3):699-707.

5. Hsieh $\mathrm{CH}$, et al. Circulating epithelial cell enumeration facilitates the identification and follow-up of a patient with early stage papillary thyroid microcarcinoma: A case report. Clin Chim Acta. 2016;454:107-11.

6. Ilie M, et al. Clinical value of circulating endothelial cells and of soluble CD146 levels in patients undergoing surgery for non-small cell lung cancer. Br J Cancer. 2014;110(5):1236-43.

7. Lin JD, et al. Circulating Epithelial Cell Characterization and Correlation with Remission and Survival in Patients with Thyroid Cancer. Thyroid. 2018;28(11):1479-89.

8. Beije $\mathrm{N}$, et al. Prognostic value and kinetics of circulating endothelial cells in patients with recurrent glioblastoma randomised to bevacizumab plus lomustine, bevacizumab single agent or lomustine 
single agent. A report from the Dutch Neuro-Oncology Group BELOB trial. Br J Cancer. 2015;113(2):226-31.

9. Li Y, et al. Evolutionary Expression of HER2 Conferred by Chromosome Aneuploidy on Circulating Gastric Cancer Cells Contributes to Developing Targeted and Chemotherapeutic Resistance. Clin Cancer Res. 2018;24(21):5261-71.

10. Wang L, et al. Quantified postsurgical small cell size CTCs and EpCAM(+) circulating tumor stem cells with cytogenetic abnormalities in hepatocellular carcinoma patients determine cancer relapse. Cancer Lett. 2018;412:99-107.

11. Lin PP, et al. Comprehensive in situ co-detection of aneuploid circulating endothelial and tumor cells. Sci Rep. 2017;7(1):9789.

12. Szczerba BM, et al. Neutrophils escort circulating tumour cells to enable cell cycle progression. Nature. 2019;566(7745):553-7.

13. Garcia-Romero N, et al. Extracellular vesicles compartment in liquid biopsies: Clinical application. Mol Aspects Med. 2018;60:27-37.

14. Kai K, Dittmar RL, Sen S. Secretory microRNAs as biomarkers of cancer. Semin Cell Dev Biol. 2018;78:22-36.

15. Best MG, Wesseling P, Wurdinger T. Tumor-Educated Platelets as a Noninvasive Biomarker Source for Cancer Detection and Progression Monitoring. Cancer Res. 2018;78(13):3407-12.

16. Zhang W, et al. Liquid Biopsy for Cancer: Circulating Tumor Cells, Circulating Free DNA or Exosomes? Cell Physiol Biochem. 2017;41(2):755-68.

17. Zhang J, et al. Circulating tumor cells with karyotyping as a novel biomarker for diagnosis and treatment of nasopharyngeal carcinoma. BMC Cancer. 2018;18(1):1133.

18. Li Y, et al. Aneuploidy of chromosome 8 in circulating tumor cells correlates with prognosis in patients with advanced gastric cancer. Chin J Cancer Res. 2016;28(6):579-88.

19. Hida K, et al. Tumor-associated endothelial cells with cytogenetic abnormalities. Cancer Res. 2004;64(22):8249-55.

20. Akino T, et al. Cytogenetic abnormalities of tumor-associated endothelial cells in human malignant tumors. Am J Pathol. 2009;175(6):2657-67.

21. Kondoh $\mathrm{M}$, et al. Hypoxia-induced reactive oxygen species cause chromosomal abnormalities in endothelial cells in the tumor microenvironment. PLoS One. 2013;8(11):e80349.

22. Yuan DM, et al. Predictive and prognostic significance of circulating endothelial cells in advanced non-small cell lung cancer patients. Tumour Biol. 2015;36(11):9031-7.

23. Zahran AM, et al. Circulating endothelial cells and their progenitors in acute myeloid leukemia. Oncol Lett. 2016;12(3):1965-70.

24. Zhao R, et al. Endothelial cells promote metastasis of prostate cancer by enhancing autophagy. J Exp Clin Cancer Res. 2018;37(1):221. 
25. Wohlfeil SA, et al. Hepatic Endothelial Notch Activation Protects against Liver Metastasis by Regulating Endothelial-Tumor Cell Adhesion Independent of Angiocrine Signaling. Cancer Res. 2019;79(3):598-610.

26. Zhao Y, et al., Tumor biology and multidisciplinary strategies of oligometastasis in gastrointestinal cancers. Semin Cancer Biol, 2019.

27. Zhang L, et al., PD-L1(+) aneuploid circulating tumor endothelial cells exhibit resistance to the checkpoint blockade immunotherapy in advanced NSCLC patients. Cancer Lett, 2019.

\section{Table}

Table 1. Clinical characteristics of 98 enrolled patients with SCLC

\begin{tabular}{ll}
\cline { 1 - 1 } N (percentage, \%) & \multicolumn{1}{c}{$60(43-84)$} \\
Age (years, median, range) & \\
Male & $58 \square 59.18 \% \square$ \\
Female & $40 \square 40.82 \% \square$ \\
Disease stage & \\
Normal & $18 \square 18.36 \% \square$ \\
Benign & $22 \square 22.45 \% \square$ \\
Early & $34 \square 34.69 \% \square$ \\
Late & $24 \square 24.49 \% \square$ \\
\hline
\end{tabular}




\section{figure1}

A

стC

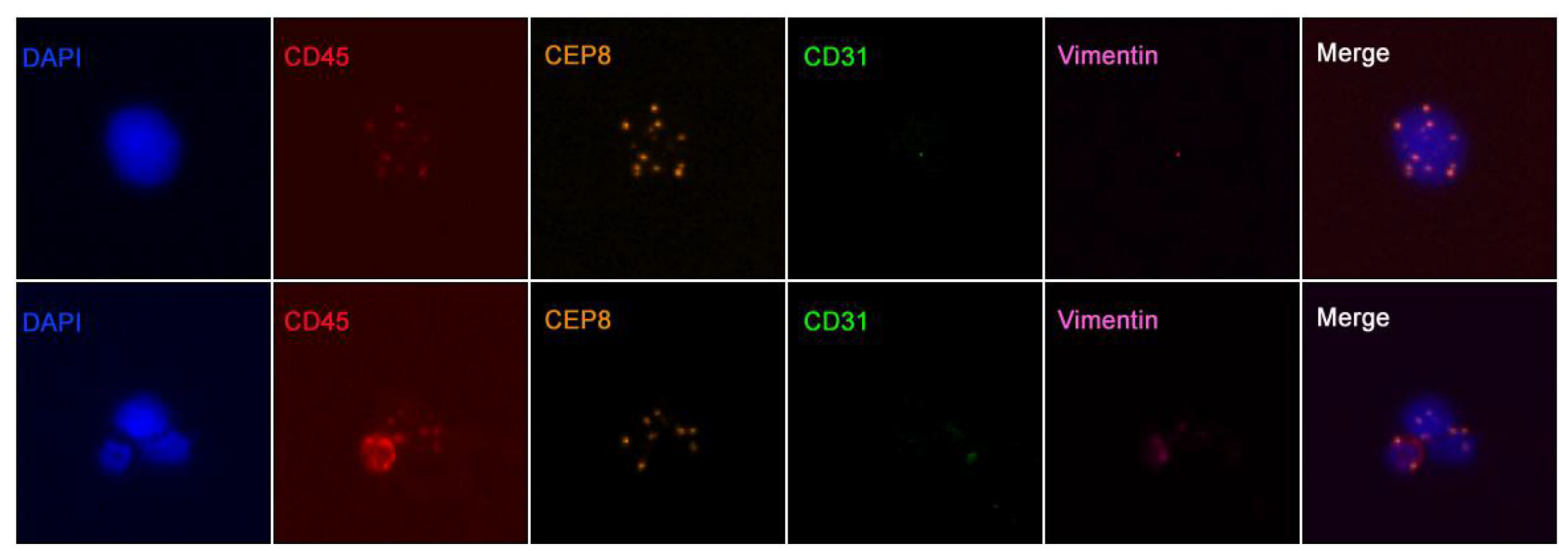

B

CTEC

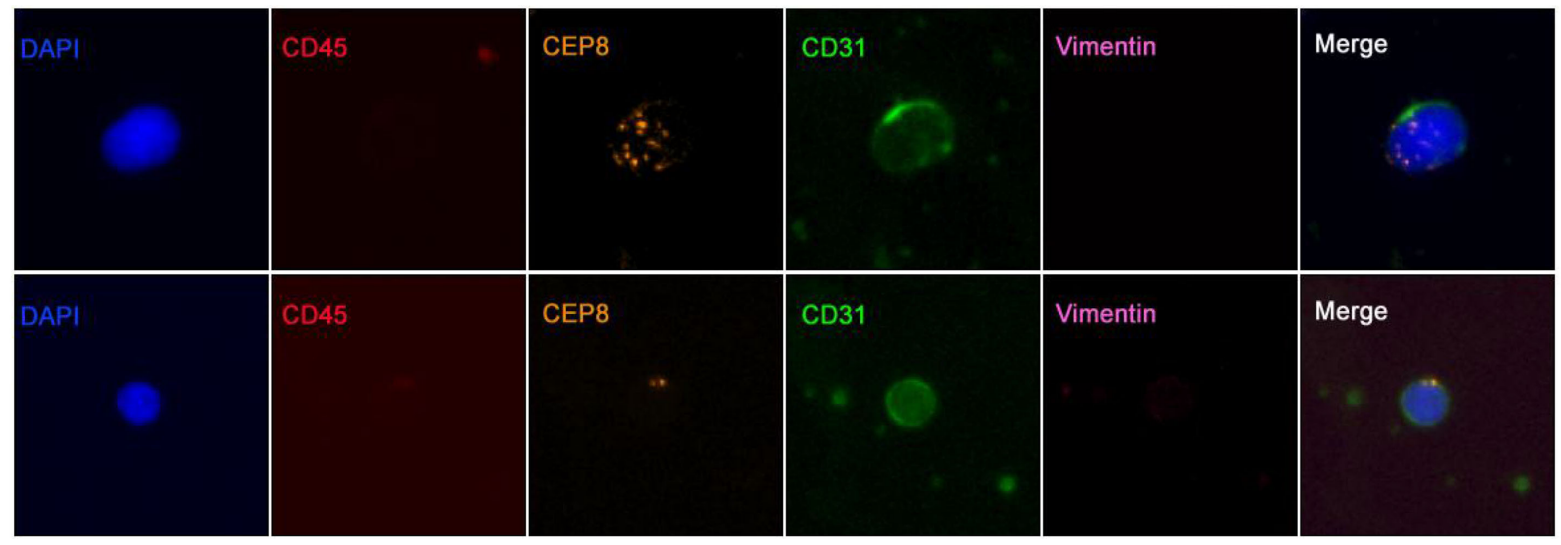

Figure 1

Detection of CTC and CTEC in peripheral blood of patients with non-small cell lung cancer by SE-iFISH (A). The two representative CTC images found by the SE-iFISH method. the CTC is DAPI+/CD45-/CD31-/CEP8+, vimentin represents one of the tumor markers, either positive or negative. (B). The two representative CTEC images found by the SE-iFISH method. the CTEC is $\mathrm{DAPI}+/ \mathrm{CD} 45-/ C D 31+/ C E P 8+$, vimentin represents one of the tumor markers, either positive or negative. 
figure 2

A

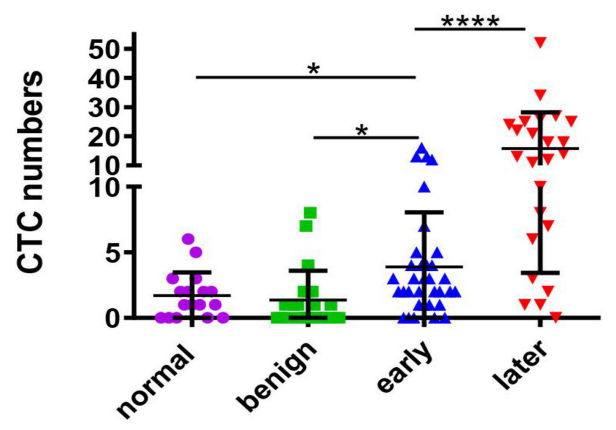

C

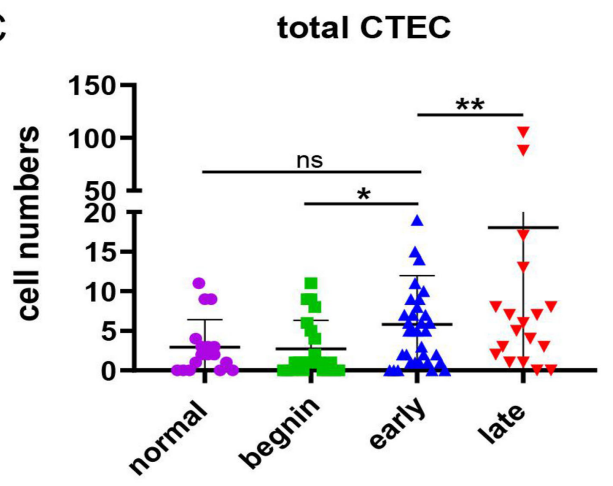

$\mathrm{B}$

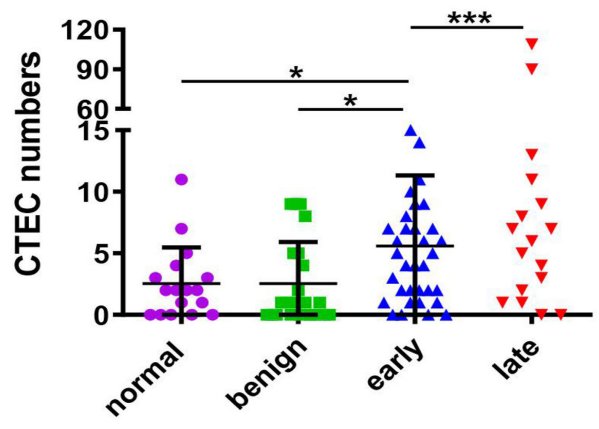

$\mathrm{D}$

CTC+CTEC

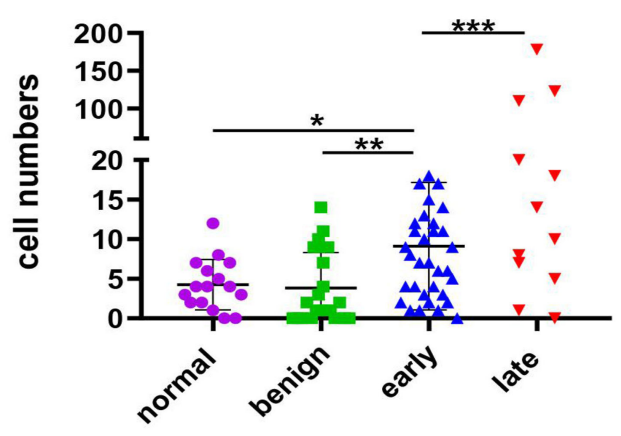

E correlation

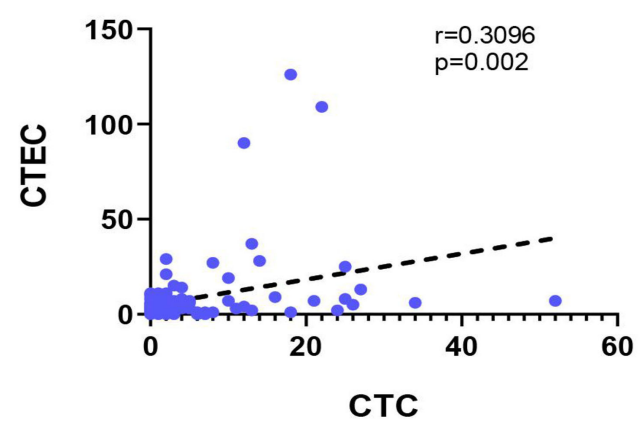

Figure 3

Changes in total CTC and CTEC numbers at different stages of lung cancer development (A). Changes in total CTC counts in normal, benign nodules and patients with early and advanced lung cancer; (B).

Changes in total aneuploid CTEC counts in normal, benign nodules and patients with early and advanced lung cancer; (C). Changes in total aneuploid and diploid CTEC counts in normal, benign nodules and patients with early and advanced lung cancer; (D). Changes in the overall number of CTC and CTEC in 
normal, benign nodules, and patients with early and advanced lung cancer; $(E)$. Correlation between the dynamics of CTC and CTEC in numbers. Data are presented as the mean $\pm S D,{ }^{*} p<0.05,{ }^{*} p<0.01$, ${ }^{\star \star *} p<$ $0.001, * \star \star \star p<0.0001$. blank means no significance.

\section{figure 3}

A

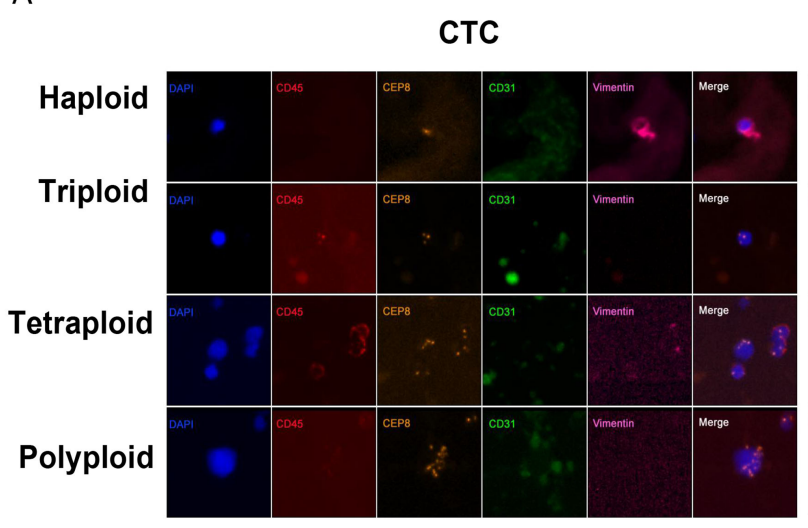

CEC

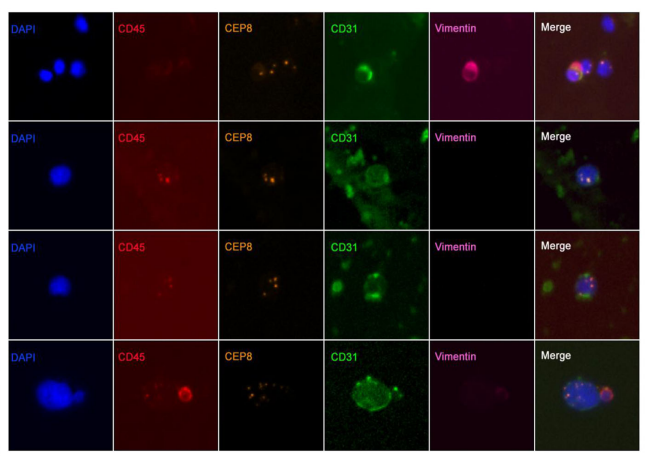

B

C

CTC
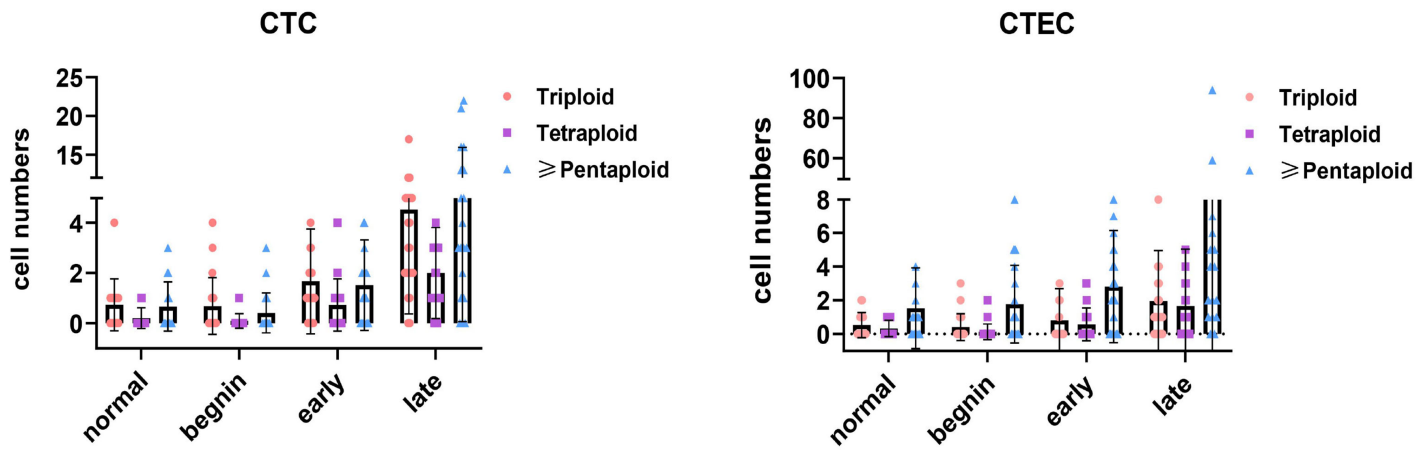

E
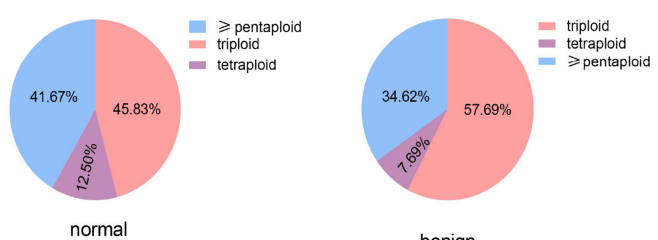

benign

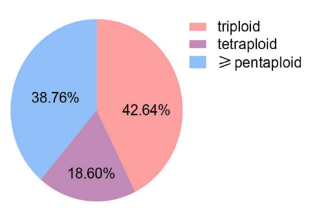

early

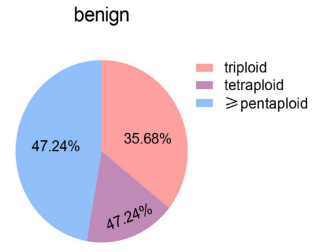

late

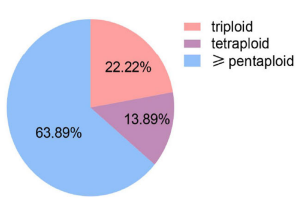

normal

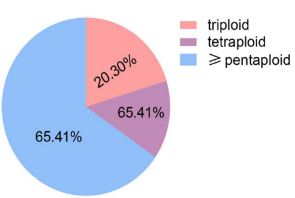

early

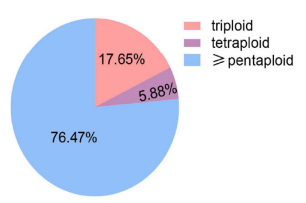

benign

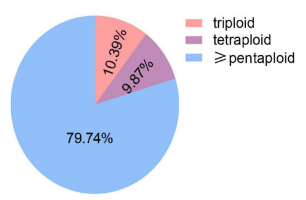

late

\section{Figure 5}

Haploid distribution of aneuploid CTC and CTEC in different populations (A). Schematic diagram of CTC and CTEC for different chromosome ploidies detected by the SE-iFISH platform; (B and D). Scatter plots 
and pie charts show the differences in the distribution of different chromosomal ploidy of CTCs in each subgroup (normal, benign nodules, early lung cancer, and advanced lung cancer); ( $C$ and $E)$. Scatter plots and pie charts show the differences in the distribution of different chromosomal ploidy of CTECs in each subgroup (normal, benign nodules, early lung cancer, and advanced lung cancer); Data are presented as the mean $\pm S D,{ }^{*} p<0.05,{ }^{* \star} p<0.01,{ }^{* \star *} p<0.001,{ }^{* \star * \star} p<0.0001$. blank means no significance.

figure 4
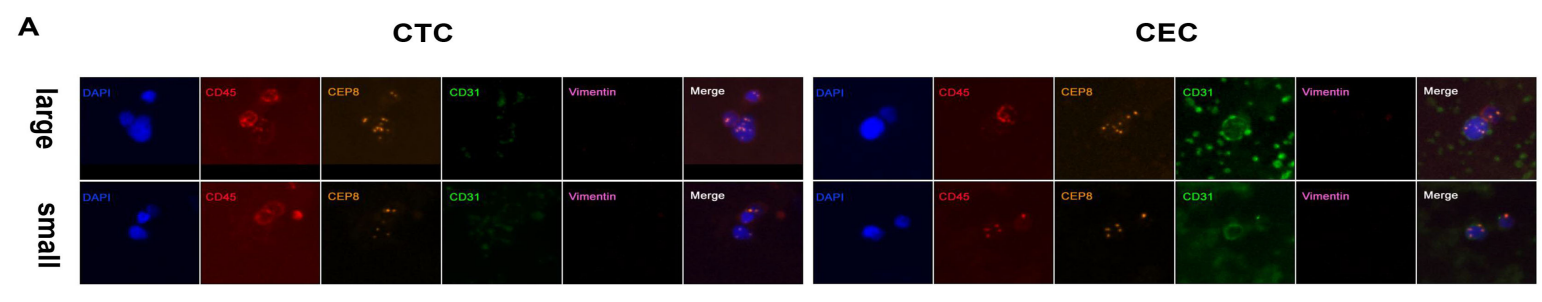

B

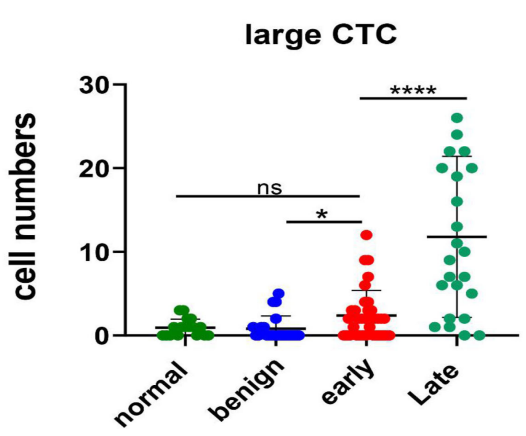

D

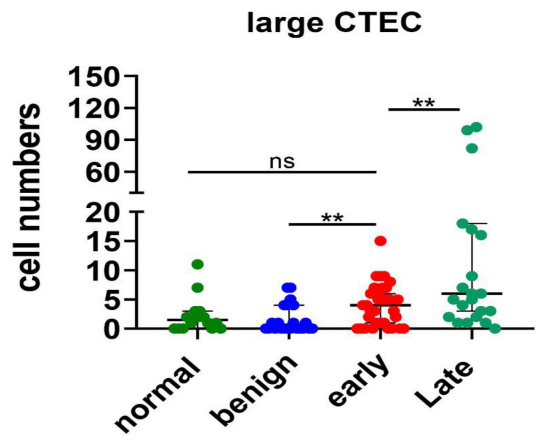

C

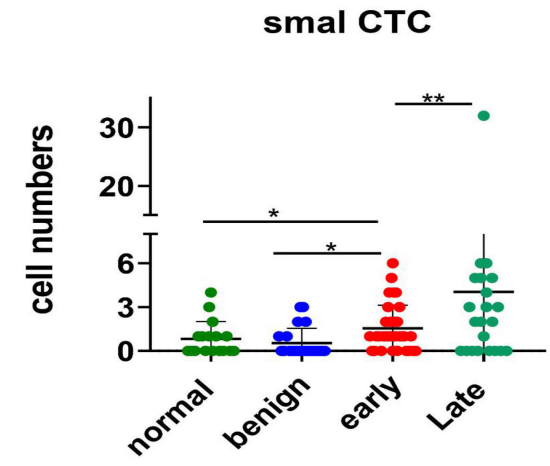

E

small CTEC

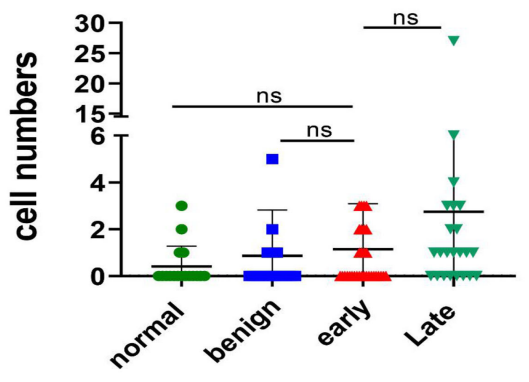

$\mathbf{F}$

large cells

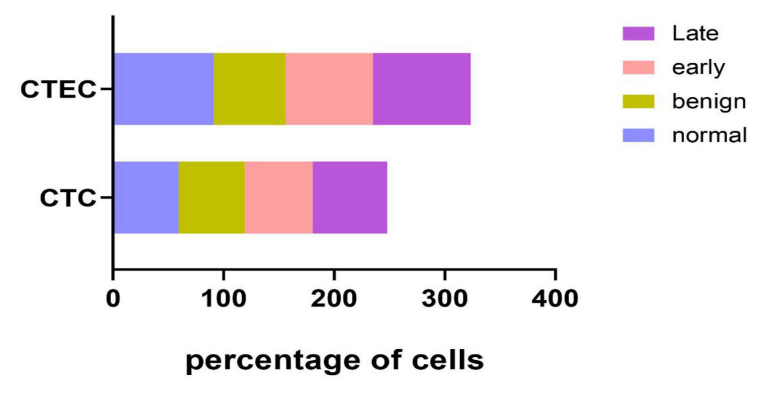

Figure 7 
Differences in the number distribution of large and small CTC and CTEC in different populations (A). Representative pictures of large ( $\geq 5 \mathrm{um}$ ) and small ( $<5 \mathrm{um}$ ) CTC and CTEC; (B). Large CTC quantity difference among four categories of population; (C). Small CTC quantity difference among four categories of population; (D). Large CTEC quantity difference among four categories of population; (E). Small CTEC quantity difference among four categories of population; $(F)$. Histogram represents the proportion of large cells (CTC and CTEC) in different populations; Data are presented as the mean \pm SD, ${ }^{\star} p<0.05,{ }^{\star \star} p<0.01,{ }^{\star \star \star} p<0.001, * \star \star \star p<0.0001$. blank means no significance.

figure 5

A

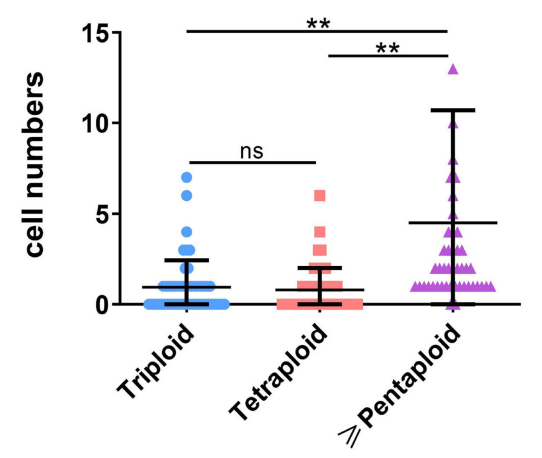

C

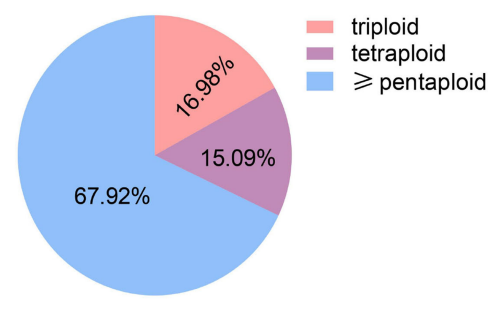

large CTC

E

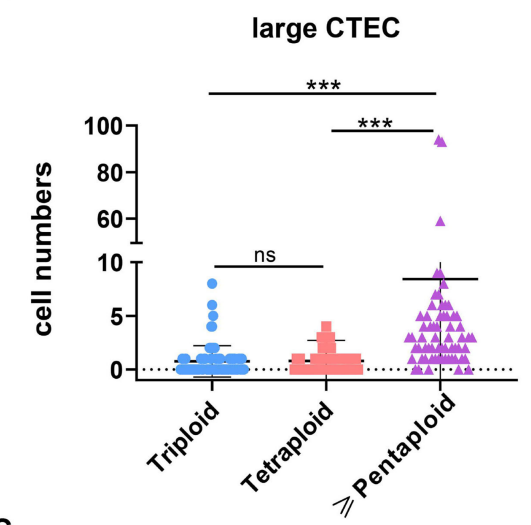

G

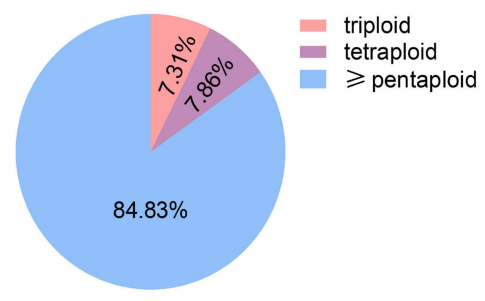

large CEC
B

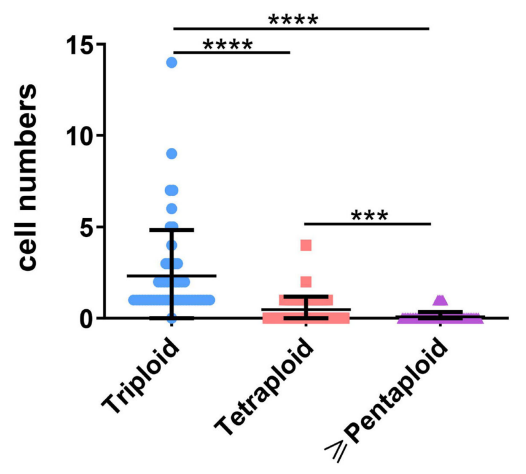

D

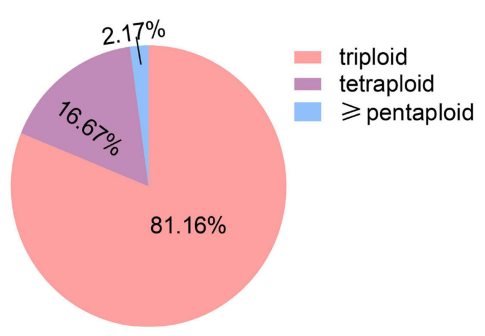

small CTC
F

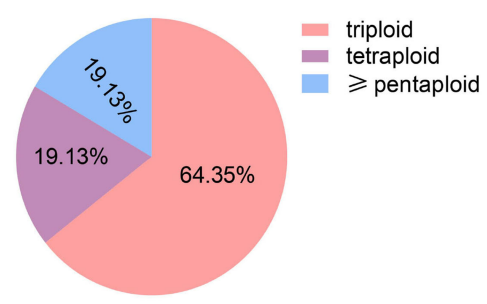

small CEC

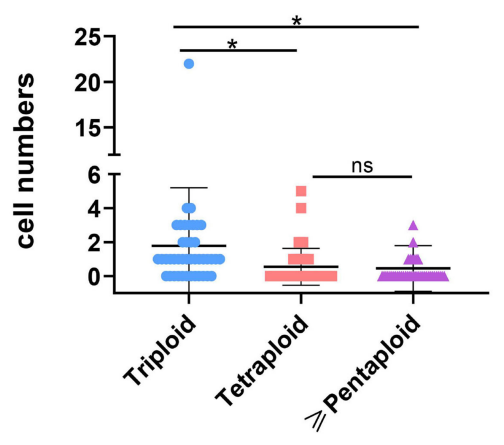

Page $21 / 24$ 
Figure 9

Chromosome ploidy differences between CTC and CTEC of different sizes (A and E). Chromosome ploidy distribution of large CTC; (B and F). Chromosome ploidy distribution of small CTC; (C and G). Chromosome ploidy distribution of large CTEC; (D and H). Chromosome ploidy distribution of small CTEC.

A

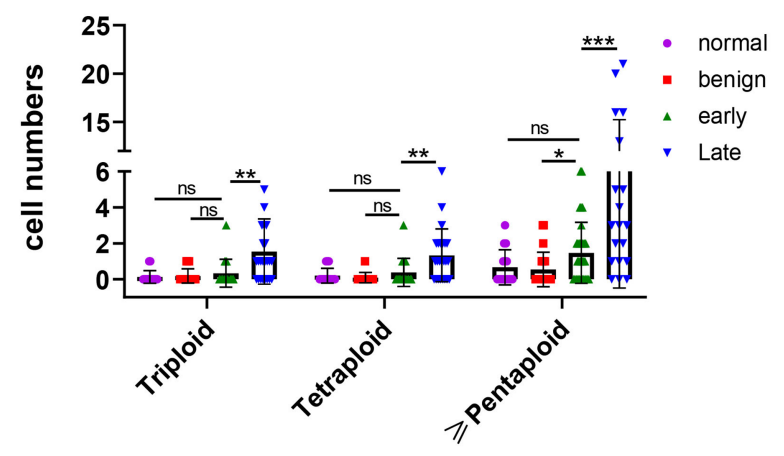

C

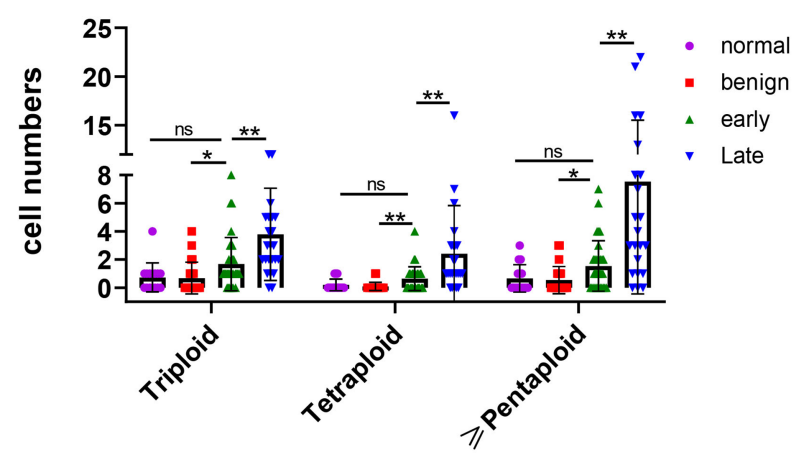

E

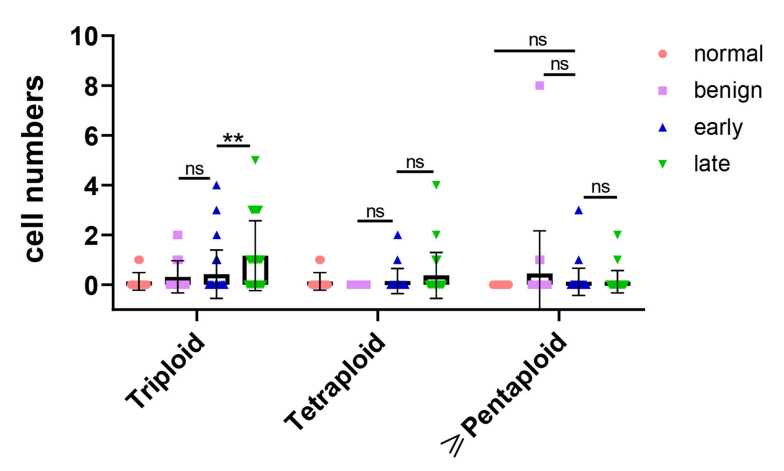

B

small CTC

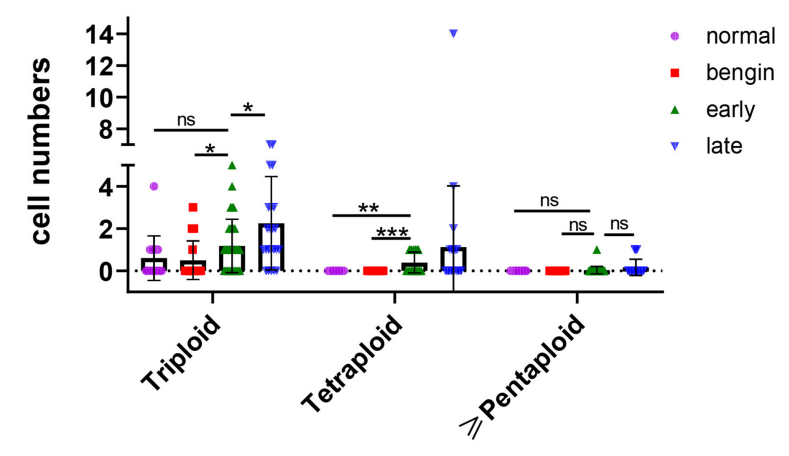

D

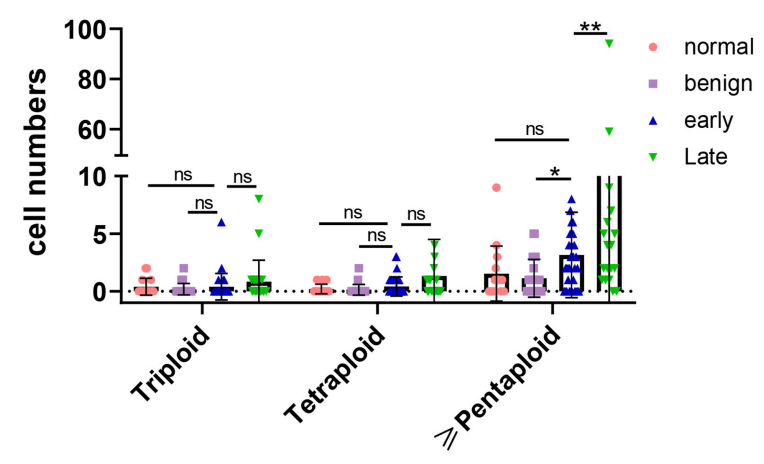

F

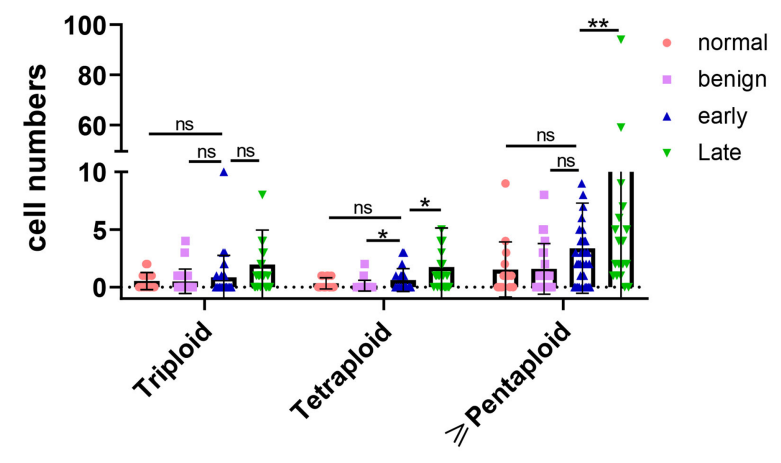


CTC and CTEC size and chromosome ploidy differences in four different populations (A). Distribution of chromosomal ploidy of large CTC in different populations; (B). Distribution of chromosomal ploidy of small CTC in different populations; (C). Distribution of chromosomal ploidy of total CTC in different populations; (D). Distribution of chromosomal ploidy of large CTEC in different populations; (E). Distribution of chromosomal ploidy of small CTEC in different populations; $(F)$. Distribution of chromosomal ploidy of total CTC in different populations; Data are presented as the mean $\pm S D,{ }^{*}<0.05$, $\star \star p<0.01,{ }^{* \star *} p<0.001, * \star \star * p<0.0001$. blank means no significance.

\section{figure 7}

A

\section{Isolation phase cell}

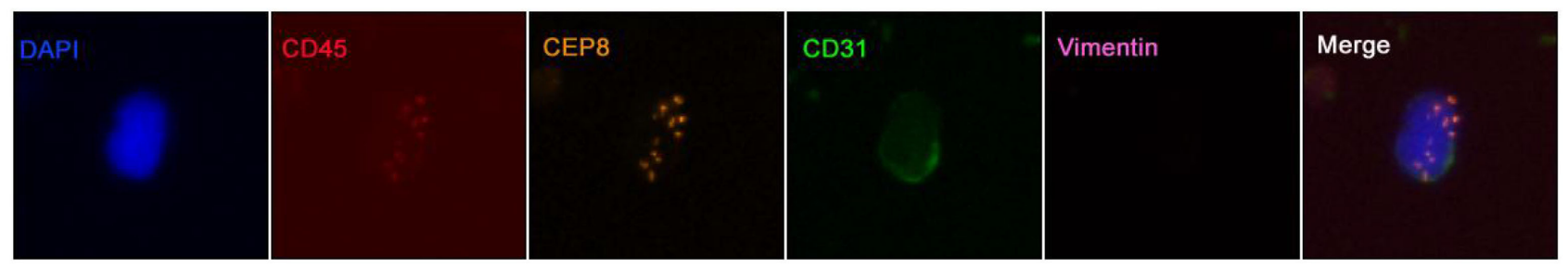

B

CTEC clusters
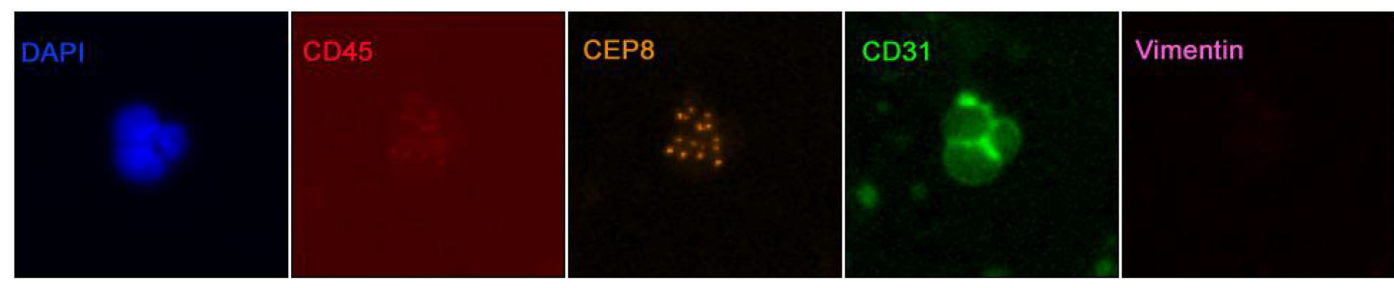

Merge

C

clusters of CTEC and white cell

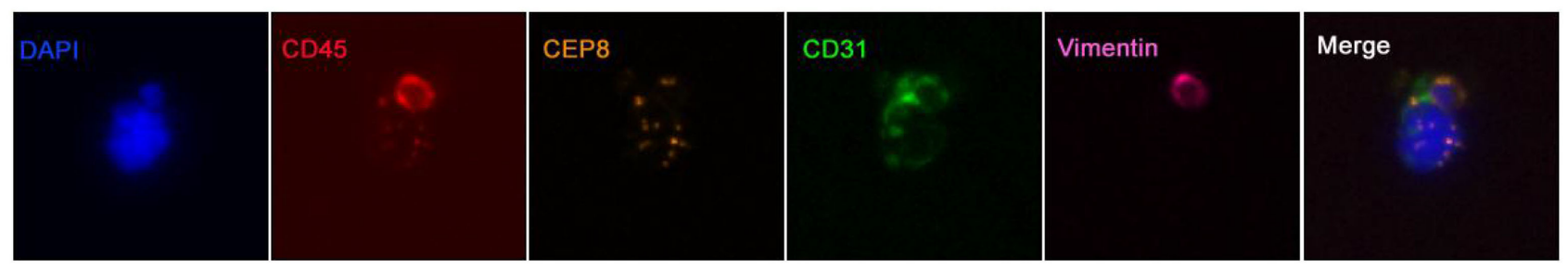

D

vim+ CTEC
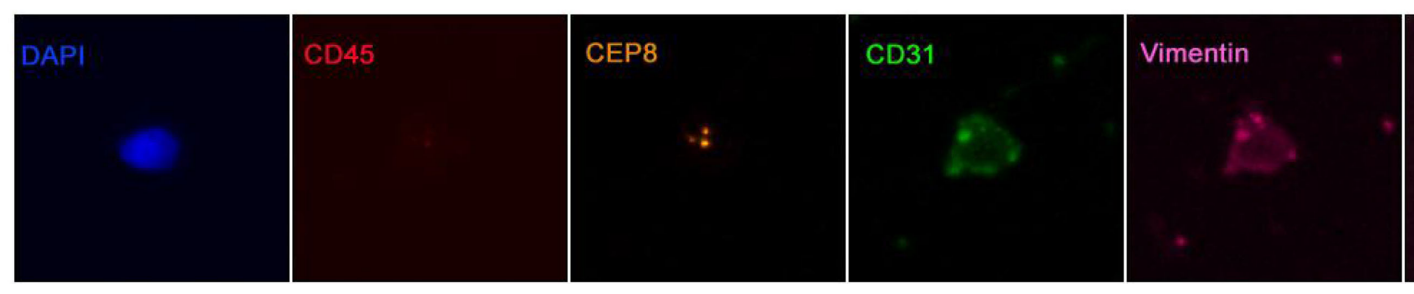

Merge

Figure 13 
Other biological characteristics of CTEC (A). Aneuploid CTEC in division; (B). Cell clumps of aneuploid CTEC; (C). Cell clumps formed by aneuploid CTEC and leukocytes; (D). vimentin-positive aneuploid CTEC. 